\title{
The importance of early treatment with tranexamic acid in bleeding trauma patients: an exploratory analysis of the CRASH-2 randomised controlled trial
}

\author{
The CRASH-2 collaborators*
}

\section{Summary}

Background The aim of the CRASH-2 trial was to assess the effects of early administration of tranexamic acid on death, vascular occlusive events, and blood transfusion in trauma patients with significant haemorrhage. Tranexamic acid significantly reduced all-cause mortality. Because tranexamic acid is thought to exert its effect through inhibition of fibrinolysis, we undertook exploratory analyses of its effect on death due to bleeding.

Methods The CRASH-2 trial was undertaken in 274 hospitals in 40 countries. 20211 adult trauma patients with, or at risk of, significant bleeding were randomly assigned within $8 \mathrm{~h}$ of injury to either tranexamic acid (loading dose $1 \mathrm{~g}$ over $10 \mathrm{~min}$ followed by infusion of $1 \mathrm{~g}$ over $8 \mathrm{~h}$ ) or placebo. Patients were randomly assigned by selection of the lowest numbered treatment pack from a box containing eight numbered packs that were identical apart from the pack number. Both participants and study staff (site investigators and trial coordinating centre staff) were masked to treatment allocation. We examined the effect of tranexamic acid on death due to bleeding according to time to treatment, severity of haemorrhage as assessed by systolic blood pressure, Glasgow coma score (GCS), and type of injury. All analyses were by intention to treat. The trial is registered as ISRCTN86750102, ClinicalTrials.gov NCT00375258, and South African Clinical Trial Register/Department of Health DOH-27-0607-1919.

Findings 10096 patients were allocated to tranexamic acid and 10115 to placebo, of whom 10060 and 10067 , respectively, were analysed. 1063 deaths $(35 \%)$ were due to bleeding. We recorded strong evidence that the effect of tranexamic acid on death due to bleeding varied according to the time from injury to treatment (test for interaction $\mathrm{p}<0.0001$ ). Early treatment ( $\leq 1 \mathrm{~h}$ from injury) significantly reduced the risk of death due to bleeding $(198 / 3747$ [5.3\%] events in tranexamic acid group vs $286 / 3704$ [7.7\%] in placebo group; relative risk [RR] 0.68 , 95\% CI $0.57-0.82 ; \mathrm{p}<0.0001)$. Treatment given between 1 and $3 \mathrm{~h}$ also reduced the risk of death due to bleeding $(147 / 3037$ [4.8\%] vs 184/2996 [6.1\%]; RR 0.79, 0.64-0.97; $\mathrm{p}=0.03)$. Treatment given after $3 \mathrm{~h}$ seemed to increase the risk of death due to bleeding (144/3272 [4.4\%] vs 103/3362 [3.1\%]; RR 1.44, 1.12-1·84; p=0 004). We recorded no evidence that the effect of tranexamic acid on death due to bleeding varied by systolic blood pressure, Glasgow coma score, or type of injury.

Interpretation Tranexamic acid should be given as early as possible to bleeding trauma patients. For trauma patients admitted late after injury, tranexamic acid is less effective and could be harmful.

Funding UK NIHR Health Technology Assessment programme, Pfizer, BUPA Foundation, and J P Moulton Charitable Foundation.

\section{Introduction}

The CRASH-2 trial showed that administration of tranexamic acid to adult trauma patients with, or at risk of, significant haemorrhage, within $8 \mathrm{~h}$ of injury, significantly reduces all-cause mortality (relative risk $[\mathrm{RR}]$ 0 $91,95 \%$ CI 0 $85-0 \cdot 97 ; \mathrm{p}=0 \cdot 0035$ ) with no apparent increase in vascular occlusive events. ${ }^{1}$ As a consequence of this trial, tranexamic acid has been incorporated into trauma treatment protocols worldwide.

Results from the CRASH-2 trial raise some important questions. The trial was motivated by the evidence that tranexamic acid reduces bleeding in patients undergoing elective surgery, and the hypothesised mechanism was inhibition of fibrinolysis leading to improved effectiveness of haemostasis. ${ }^{2}$ However, no significant difference was recorded in transfusion requirements between the tranexamic acid and placebo groups, and the CRASH-2 trial did not measure the effect of this drug on fibrinolytic assays. Thus an alternative hypothesis is that tranexamic acid might act by reducing the pro-inflammatory effects of plasmin, rather than by improving haemostasis. ${ }^{3}$

There has also been discussion about which trauma patients should be treated with tranexamic acid. The CRASH-2 trial ${ }^{1}$ reported the few subgroup analyses that were prespecified in the statistical analysis plan. These analyses assessed the effect of tranexamic acid on the primary endpoint of all-cause mortality, according to time since injury, systolic blood pressure, Glasgow coma score, and type of injury. No strong evidence of
Published Online March 24, 2011 DOI:10.1016/S01406736(11)60278-X

See Online/Comment DOI:10.1016/S0140 6736(11)60396-6 See Online/Correspondence DOI:10.1016/S01406736(11)60317-6

${ }^{*}$ Members listed at end of Article and in online version of this paper Correspondence to: Clinical Trials Unit, London School of Hygiene and Tropical Medicine, London WC1E 7HT, UK crash@lshtm.ac.uk 
heterogeneity was recorded for any of these analyses, suggesting that tranexamic acid is likely to be equally effective in all the subgroups examined.

The focus on all-cause mortality was appropriate because it is an outcome that matters to patients and one that is not affected by the methodological problem of

\begin{tabular}{|c|c|c|c|c|}
\hline & $\mathrm{N}$ & All causes of death & Bleeding death & Non-bleeding death \\
\hline Overall & 20127 & $\begin{array}{l}0.91(0.85-0.97) \\
p=0.0035\end{array}$ & $\begin{array}{l}0.85(0.76-0.96) ; \\
\mathrm{p}=0.0077\end{array}$ & $\begin{array}{l}0.94(0.86-1.02) ; \\
p=0.13\end{array}$ \\
\hline \multicolumn{5}{|l|}{ Time to treatment (h) } \\
\hline$\leq 1$ & 7451 & $0.87(0.76-0.97)$ & $0.68(0.57-0.82)$ & $1.04(0.89-1.21)$ \\
\hline$>1-3$ & 6033 & $0.87(0.77-0.97)$ & $0.79(0.64-0.97)$ & $0.91(0.78-1.05)$ \\
\hline$>3$ & 6634 & $1.00(0.90-1.13)$ & $1.44(1.12-1.84)$ & $0.89(0.78-1.02)$ \\
\hline$\chi^{2}$ test of homogeneity & & $4.411(p=0.11)$ & $23.516(p=0.0000)$ & $2.537(p=0.28)$ \\
\hline
\end{tabular}

Table 1: Relative risk $(95 \% \mathrm{Cl})$ of death with tranexamic acid, overall and by time to treatment

\begin{tabular}{|c|c|c|c|}
\hline & $\leq 1 \mathrm{~h}(\mathrm{n}=7451)$ & $>1-3 \mathrm{~h}(\mathrm{n}=6033)$ & $>3 \mathrm{~h}(\mathrm{n}=6634)$ \\
\hline \multicolumn{4}{|l|}{ Age (years) } \\
\hline Mean (SD) & $33 \cdot 4(13 \cdot 9)$ & $35 \cdot 0(14.0)$ & $35 \cdot 5(14 \cdot 8)$ \\
\hline$<25$ & $2283(30 \cdot 6 \%)$ & $1557(25 \cdot 8 \%)$ & $1773(26 \cdot 7 \%)$ \\
\hline $25-34$ & $2360(31 \cdot 7 \%)$ & $1832(30 \cdot 4 \%)$ & $1882(28.4 \%)$ \\
\hline $35-44$ & $1356(18 \cdot 2 \%)$ & $1177(19 \cdot 5 \%)$ & $1262(19.0 \%)$ \\
\hline$>44$ & $1452(19 \cdot 5 \%)$ & $1467(24 \cdot 3 \%)$ & $1716(25 \cdot 9 \%)$ \\
\hline \multicolumn{4}{|l|}{ Systolic blood pressure $(\mathrm{mm} \mathrm{Hg})$} \\
\hline$\leq 75$ & $1380(18 \cdot 5 \%)$ & $1012(16 \cdot 8 \%)$ & $768(11 \cdot 6 \%)$ \\
\hline $76-89$ & $1203(16 \cdot 1 \%)$ & $1064(17 \cdot 6 \%)$ & $1029(15 \cdot 5 \%)$ \\
\hline$>89$ & $4857(65 \cdot 2 \%)$ & $3955(65 \cdot 6 \%)$ & $4821(72 \cdot 7 \%)$ \\
\hline \multicolumn{4}{|l|}{ Heart rate (beats per min) } \\
\hline$<77$ & $681(9 \cdot 1 \%)$ & $450(7 \cdot 5 \%)$ & $603(9 \cdot 1 \%)$ \\
\hline 77-91 & $1189(16 \cdot 0 \%)$ & $971(16 \cdot 1 \%)$ & $1326(20.0 \%)$ \\
\hline $92-107$ & $1888(25 \cdot 3 \%)$ & $1562(25 \cdot 9 \%)$ & $1625(24 \cdot 5 \%)$ \\
\hline$>107$ & $3637(48.8 \%)$ & $2990(49 \cdot 6 \%)$ & $3059(46 \cdot 1 \%)$ \\
\hline \multicolumn{4}{|l|}{ Respiratory rate (breaths per min) } \\
\hline$<10$ & $149(2 \cdot 0 \%)$ & $82(1 \cdot 4 \%)$ & $77(1 \cdot 2 \%)$ \\
\hline $10-29$ & $6144(82 \cdot 5 \%)$ & $4992(82 \cdot 7 \%)$ & $5590(84 \cdot 3 \%)$ \\
\hline$>29$ & $1077(14 \cdot 5 \%)$ & $901(14.9 \%)$ & $923(13.9 \%)$ \\
\hline \multicolumn{4}{|l|}{ Capillary refill time (s) } \\
\hline$\leq 2$ & $2450(32 \cdot 9 \%)$ & $2140(35 \cdot 5 \%)$ & $2217(33 \cdot 4 \%)$ \\
\hline $3-4$ & $3472(46 \cdot 6 \%)$ & $2773(46 \cdot 0 \%)$ & $3110(46 \cdot 9 \%)$ \\
\hline$>4$ & $1131(15 \cdot 2 \%)$ & $963(16 \cdot 0 \%)$ & $1257(19 \cdot 0 \%)$ \\
\hline \multicolumn{4}{|l|}{ Glasgow coma score } \\
\hline Severe (3-8) & $1000(13 \cdot 4 \%)$ & $1124(18.6 \%)$ & $1494(22 \cdot 5 \%)$ \\
\hline Moderate (9-12) & $868(11 \cdot 7 \%)$ & $915(15 \cdot 2 \%)$ & $909(13 \cdot 7 \%)$ \\
\hline Mild (13-15) & $5577(74 \cdot 9 \%)$ & $3994(66 \cdot 2 \%)$ & $4214(63 \cdot 5 \%)$ \\
\hline \multicolumn{4}{|l|}{ Continents } \\
\hline Asia & $1213(16 \cdot 3 \%)$ & $2475(41 \cdot 0 \%)$ & $3656(55 \cdot 1 \%)$ \\
\hline Africa & $2490(33 \cdot 4 \%)$ & $1437(23 \cdot 8 \%)$ & $872(13 \cdot 1 \%)$ \\
\hline Central and South America & $2453(32 \cdot 9 \%)$ & $1456(24 \cdot 1 \%)$ & $1355(20 \cdot 4 \%)$ \\
\hline North America, Europe, and Oceania & $1295(17 \cdot 4 \%)$ & $665(11 \cdot 0 \%)$ & $751(11 \cdot 3 \%)$ \\
\hline
\end{tabular}

\section{Methods}

\section{Study design and patients}

The background to the trial, methods, and baseline characteristics of the randomised patients have been previously reported. ${ }^{1}$ Briefly, we randomly allocated 20211 adult trauma patients with, or at risk of, significant bleeding who were within $8 \mathrm{~h}$ of injury to either tranexamic acid (loading dose $1 \mathrm{~g}$ over $10 \mathrm{~min}$ followed by infusion of $1 \mathrm{~g}$ over $8 \mathrm{~h}$ ) or matching placebo, with $99.6 \%$ follow-up. In most hospitals we used a local pack system for randomisation. After eligibility had been confirmed and the locally approved consent procedures had been completed, patients were randomly assigned by selection of the lowest numbered treatment pack from a box containing eight numbered packs. Apart from the pack number, the treatment packs were identical. The pack number was recorded on the entry form, which was sent to the Trial Coordinating Centre in London, UK. Hospitals with telephone access used a telephone randomisation service. Both participants and study staff (site investigators and trial coordinating centre staff) were masked to treatment allocation.

\section{Statistical analysis}

The primary outcome was death in hospital within 4 weeks of injury, with cause of death described with the following categories: bleeding, vascular occlusion (myocardial infarction, stroke, and pulmonary embolism), multiorgan failure, head injury, and other.

All analyses were by intention to treat. We examined the effect of the trial treatment on death due to bleeding subdivided by four baseline characteristics: (1) time from injury to treatment $(\leq 1,>1-3,>3 \mathrm{~h})$; (2) severity of haemorrhage as assessed by systolic blood pressure $(\leq 75$, 76-89, >89 mm Hg); (3) Glasgow coma score (severe 3-8, moderate 9-12, mild 13-15); and (4) type of injury (penetrating only, blunt plus blunt and penetrating). These were the same subgroup analyses that were reported previously, but for the outcome of death due to bleeding rather than for all-cause mortality.

Heterogeneity in treatment effects across subgroups was assessed by a $\chi^{2}$ test. We had prespecified that unless there was strong evidence against the null hypothesis of homogeneity of effects (ie, $\mathrm{p}<0 \cdot 001$ ), the overall RR would be considered the most reliable guide to the approximate RRs in all subgroups. To test the 


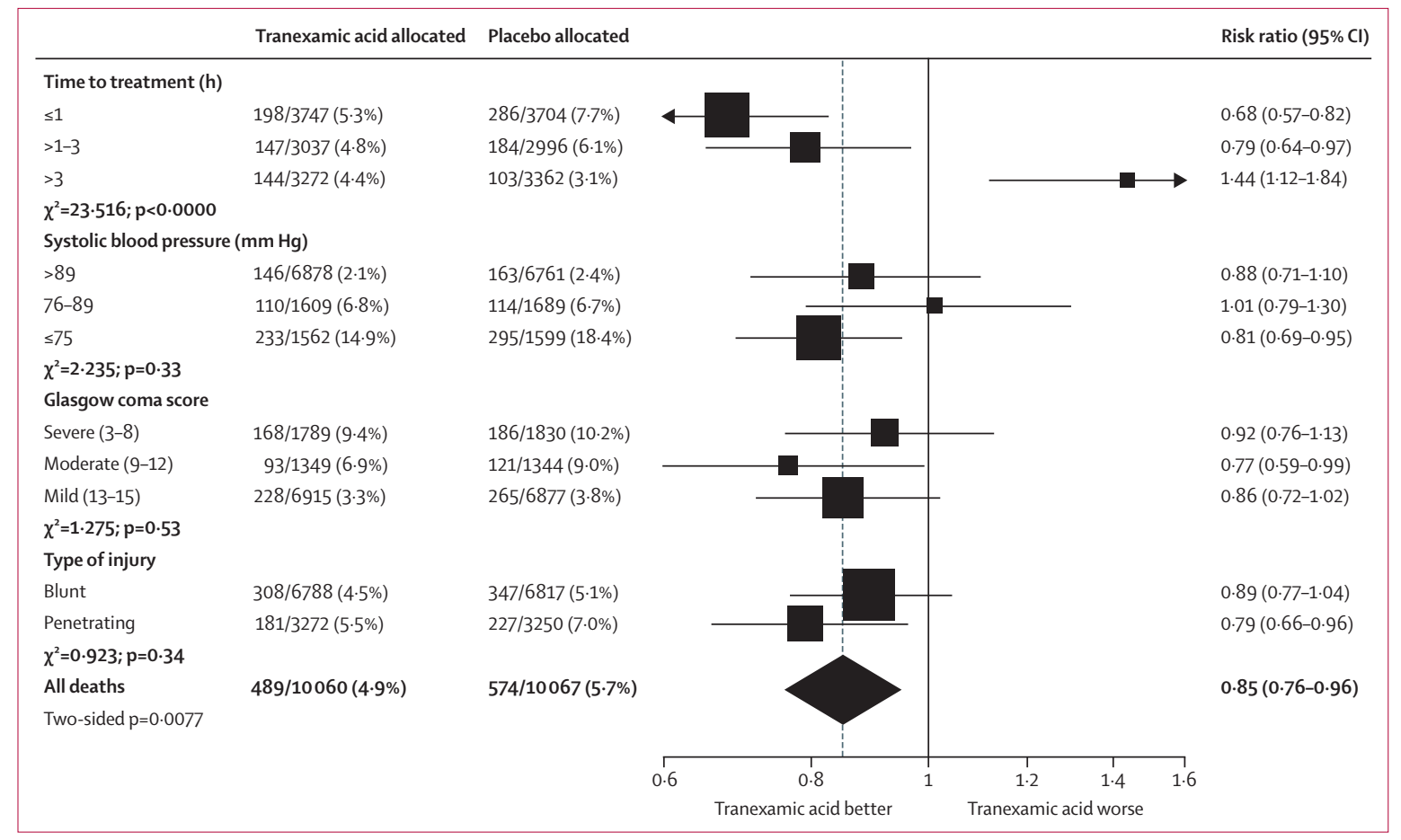

Figure 1: Mortality due to bleeding by subgroups

independence of any observed treatment interactions we ran a logistic model including all possible interactions in the four prespecified baseline characteristics and treatment subgroups.

A logistic regression was estimated with death due to bleeding as the dependent variable and treatment group and time to treatment as explanatory factors. We included an interaction parameter to allow for a proportional change in the odds ratio (OR) as time to treatment increases. ORs and $95 \% \mathrm{CIs}$ were estimated for different times to treatment. CIs were calculated with a logistic model with time as a continuous term and an interaction term between time and tranexamic acid. We also ran a model with an interaction term for time to treatment squared to allow for a non-constant proportional change in the OR.

The trial is registered as ISRCTN86750102, ClinicalTrials.gov NCT00375258, and South African Clinical Trial Register/Department of Health DOH-270607-1919.

\section{Role of the funding source}

The sponsor of the study had no role in study design, data collection, data analysis, data interpretation, or writing of the report. The corresponding author (IR) had full access to all the data in the study and had final responsibility for the decision to submit for publication.

\section{Results}

Of the 3076 deaths from all causes, death due to bleeding accounted for 1063 (35\%). The risk of death due to bleeding was significantly reduced with tranexamic acid. 489 of 10060 (4.9\%) patients died because of bleeding in the tranexamic acid group versus 574 of 10067 (5.7\%) in the placebo group (RR $0.85,95 \%$ CI $0.76-0.96$; $\mathrm{p}=0.0077)$. We noted no significant effect on the risk of death for all other (non-bleeding) causes combined (table 1).

Table 2 shows the baseline characteristics of patients according to time to treatment. Figure 1 shows the results of the subgroup analyses for death due to bleeding. Time to treatment was unknown in nine participants. Treatment given $1 \mathrm{~h}$ or less from injury significantly reduced the risk of death due to bleeding $(198 / 3747$ [5.3\%] in tranexamic acid group vs 286/3704 [7.7\%] in placebo group; RR $0 \cdot 68,95 \%$ CI $0 \cdot 57-0 \cdot 82$; $\mathrm{p}<0 \cdot 0001)$. Treatment given between 1 and $3 \mathrm{~h}$ also reduced the risk of death due to bleeding $(147 / 3037$ [4.8\%] vs 184/2996 [6.1\%]; RR 0 · 79, 0 - 64-0.97; $\mathrm{p}=0 \cdot 03$ ). Treatment given more than $3 \mathrm{~h}$ after injury significantly increased the risk of death due to bleeding (144/3272 [4.4\%] vs 103/3362 [3 · 1\%]; RR 1.44, 1.12-1.84; $\mathrm{p}=0 \cdot 004)$. We recorded strong evidence that the effect of tranexamic acid on death due to bleeding varied according to time from injury to treatment $(\mathrm{p}<0 \cdot 0001)$. The evidence for interaction remained strong even after adjustment for interactions between the other prespecified baseline characteristics and treatment $(\mathrm{p}<0 \cdot 0001$; data not shown).

The estimated OR of tranexamic acid on death due to bleeding when given immediately after injury was 0.61 


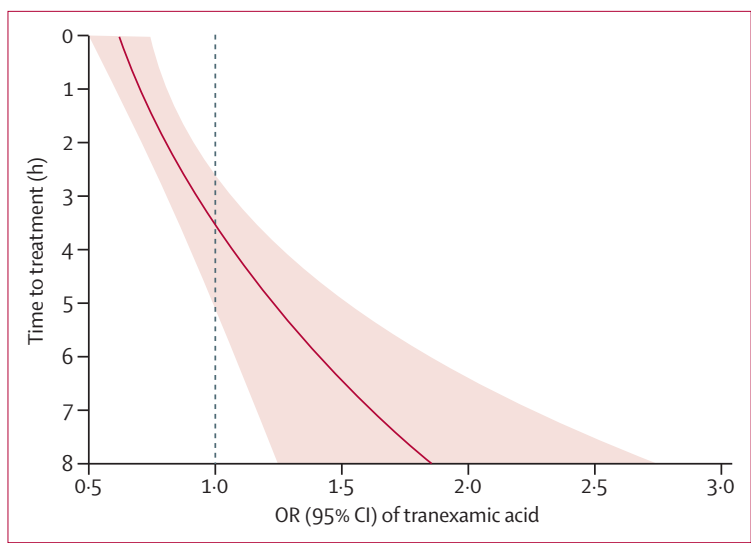

Figure 2: Effect of tranexamic acid on death due to bleeding by time to treatment

Shaded area shows $95 \% \mathrm{Cl}$. OR=odds ratio.

(95\% CI $0 \cdot 50-0 \cdot 74)$. We estimated that this OR is multiplied by 1.15 (95\% CI 1.08-1.23) for every hour that passes since the injury. Figure 2 shows how the OR and $95 \%$ CIs vary with time to treatment. The interaction term for time to treatment squared was not significant $(\mathrm{OR}=0 \cdot 99 ; \mathrm{p}=0 \cdot 38)$.

We recorded no evidence of heterogeneity for the subgroup analyses according to systolic blood pressure, Glasgow coma score at randomisation, or type of injury (figure 1). We detected no evidence of heterogeneity in the effect of tranexamic acid on the risk of non-bleeding deaths (table 1).

\section{Discussion}

The effect of tranexamic acid on death due to bleeding depends on the time between injury and onset of treatment. Early treatment with this drug seems to be much more effective than does late treatment. These results also raise the possibility that late treatment with tranexamic acid might increase the risk of death due to bleeding, although there was no evidence of any increase in all-cause mortality in patients treated after $3 \mathrm{~h}$ (table 1). This finding might indicate that patients treated with tranexamic acid beyond $3 \mathrm{~h}$ who died from bleeding might otherwise have died from some other non-bleeding cause (competing risks). If late administration does cause harm, this finding would be important since many bleeding trauma patients in lowincome and middle-income countries have long prehospital times. Indeed, about a third of trauma patients in the CRASH-2 trial were treated more than $3 \mathrm{~h}$ after the injury.

The inclusion criteria in the CRASH-2 trial were entirely clinical, and reflect the situation that doctors are faced with in clinical practice. Patients were enrolled if the treating physician judged them to have ongoing significant haemorrhage, as evidenced by hypotension or tachycardia, or if they were considered to be at risk of significant haemorrhage. Some of the included patients might not have been actively bleeding. Any such misdiagnosis would have reduced the power of the trial to show an effect of tranexamic acid on mortality from bleeding, in which case the large and highly significant reduction in bleeding mortality in patients treated with this drug within $1 \mathrm{~h}$ of injury is particularly noteworthy.

Because patients were randomly assigned soon after hospital admission, before the precise anatomical location of bleeding and other injury was known, we were unable to do a stratified analysis based on an anatomical assessment of injury severity. We acknowledge that this omission is a methodological weakness, since such an analysis might provide insight into the mechanism of action of tranexamic acid. However, since this information would not normally be available to treating clinicians, especially in view of the importance of early treatment, the clinical value of a stratified analysis based on anatomical injury severity is small.

Data for the time between injury and treatment were available for all but nine trial participants. Because in some cases the injury would not have been witnessed, this interval sometimes had to be estimated and might therefore be inaccurate. However, any inaccuracy would be independent of the trial treatment and therefore should not bias the results. The ascertainment of a death as a bleeding death might also have been inaccurate, but similarly any inaccuracy should be independent of the trial treatment.

In clinical trials, a treatment is not often beneficial in one subgroup but harmful in another (qualitative interaction), and some trialists recommend that qualitative interactions should generally be disbelieved. 5 The results of our analysis of the effect of tranexamic acid on death due to bleeding do, however, satisfy most of the criteria against which the credibility of subgroup results should be judged: ${ }^{6}$ time from injury was measured at baseline; the hypothesis that early treatment with tranexamic acid might be more effective was prespecified in the trial protocol; the interaction suggests a very low likelihood that chance explains the findings; the interaction remained significant after controlling for the non-significant interactions between treatment and the other prespecified baseline prognostic factors; the subgroup effect is large; and a biological rationale supports the interaction. Although this clinical trial was not powered to examine subgroup effects, the interaction recorded is large and highly significant.

Nevertheless, we prespecified in our trial protocol that the main subgroup analyses would be undertaken for allcause mortality, and not for mortality due to bleeding. Even though we postulated that tranexamic acid would act by reducing bleeding, we focused on all-cause mortality because overall survival is most important to patients. However, in view of the significant reduction in all-cause mortality, most of which was attributable to the effect of tranexamic acid on death due to bleeding, and the biological rationale that this drug would act by 
improving haemostasis, our analyses, although not prespecified, would seem justified.

Acute severe trauma is associated with increased fibrinolysis that contributes to an early coagulopathy and increased mortality. ${ }^{89}$ Fibrinolysis can be assessed by measurement of fibrin degradation products, which include small protein fragments called D-dimers. Brohi and colleagues ${ }^{8}$ showed that D-dimer concentrations are raised in trauma patients at the time of hospital admission (median prehospital time $28 \mathrm{~min}$ ), with the highest concentrations measured in the most severely injured patients. ${ }^{8}$ Similar results were recorded in a 2009 study from Japan that measured fibrin degradation product and D-dimers in 314 severe trauma patients..$^{10}$ If this early increased fibrinolysis exacerbates bleeding and increases the risk of death, then we might expect that an antifibrinolytic drug such as tranexamic acid would be most effective in this period.

Although we had anticipated that early treatment with tranexamic acid might be most effective, the apparent increase in the risk of death due to bleeding in patients treated more than $3 \mathrm{~h}$ after the injury is unexpected and cannot readily be explained. It could be a chance finding and there might be no real biological effect. However, patients in the late phase of trauma can develop thrombotic disseminated intravascular coagulation, and antifibrinolytics could be contraindicated in this period. ${ }^{10,11}$ Although disseminated intravascular coagulation is characterised by fibrin formation and coagulation, the rapid consumption of coagulation proteins can lead to their exhaustion, resulting in uncontrolled bleeding. The need to avoid giving an antifibrinolytic in this late phase was why we restricted trial inclusion to patients who were within $8 \mathrm{~h}$ of injury. The possibility that the change to a prothrombotic state might occur sooner than was previously expected is open to debate and needs further research. We should also bear in mind that patients who arrive at hospital many hours after injury are likely to differ from those who arrive early. For example, there could be an increased prevalence of hypothermia and acidosis. These or other differences could explain the decreased efficacy of tranexamic acid administration when given late.

A 2011 systematic review of randomised controlled trials concluded that tranexamic acid safely reduces mortality in bleeding trauma patients. ${ }^{12}$ Our results strongly endorse the importance of early administration of tranexamic acid in bleeding trauma patients and suggest that trauma systems should be configured to facilitate this recommendation (panel). In patients presenting late (several hours after injury) the clinician should be more cautious and make an assessment of the individual benefits and risks of this treatment, since the drug is likely to be much less effective and possibly even harmful. To the extent that our subgroup analyses are consistent with the results of studies showing an early
Panel: Research in context

\section{Systematic review}

A 2011 Cochrane systematic review ${ }^{12}$ of antifibrinolytic drugs for acute traumatic injury identified two randomised trials of tranexamic acid in bleeding trauma patients, involving 20451 patients. The review concluded that tranexamic acid safely reduces mortality in bleeding trauma patients without increasing the risk of adverse events.

\section{Interpretation}

Our results emphasise the importance of early administration of tranexamic acid and the need for caution in patients presenting several hours after the injury.

increased fibrinolytic coagulopathy, they support the hypothesis that tranexamic acid acts through the inhibition of fibrinolysis with improved haemostasis.

Future research using the CRASH-2 trial data will develop a prognostic model to predict death due to bleeding. ${ }^{13}$ This model will facilitate further analysis of the effect of tranexamic acid according to baseline risk of haemorrhage death.

\section{Contributors}

All members of the Writing Committee, apart from AA and GG, attended a 2-day meeting in London, UK, at which the subgroup analyses were presented and discussed and the report was drafted. Both AA and GG contributed to the discussions and drafting by phone and in correspondence.

\section{CRASH-2 trial coordination}

Writing Committee: Ian Roberts (UK) (chair), Haleema Shakur (UK), Adefemi Afolabi (Nigeria), Karim Brohi (UK), Tim Coats (UK), Yashbir Dewan (India), Satoshi Gando (Japan), Gordon Guyatt (Canada), B J Hunt (UK), Carlos Morales (Colombia), Pablo Perel (UK), David Prieto-Merino (UK), Tom Woolley (UK).

National coordinators: Jonathan Dakubo (Ghana), Tamar Gogichaishvili (Georgia), Nyoman Golden (Indonesia), Mario Izurieta (Ecuador), Hussein Khamis (Egypt), Edward Komolafe (Nigeria),

Jorge Loría-Castellanos (Mexico), Jorge Mejía-Mantilla (Colombia), Jaime Miranda (Peru), Ángeles Muñoz (Spain), Vincent Mutiso (Kenya), Patrick Okwen (Cameroon), Zulma Ortiz (Argentina), María Pascual, CENCEC (Cuba), R Ravi (India), April Roslani (Malaysia), Stefan Trenkler (Slovakia), Annalisa Volpi (Italy), Surakrant Yutthakasemsunt (Thailand).

Trial Coordinating Centre team: Ian Roberts (clinical coordinator, chief investigator), Haleema Shakur (trial manager), Pablo Perel (regional coordinator), Lin Barnetson (data manager), Maria Ramos (trial administrator), Lisa Cook (assistant trial manager, regional coordinator from 2007), Taemi Kawahara (assistant trial manager, regional coordinator from 2007), Eni Balogun (regional coordinator from 2006), Matthew Berle (trial assistant from 2007), Collette Barrow (assistant administrator from 2008), Tony Brady (programmer to 2006), Chris Rubery (data assistant from 2009), Jackie Wayte (UK nurse coordinator from 2008), Cynthia To (data assistant 2007-09). Steering Committee: Ian Franklin (chair), Brigitte Chaudhry, Tim Coats, Charles Deakin, Steve Goodacre, Beverley Hunt, David Meddings, Richard Peto, Ian Roberts, Peter Sandercock. Management Group: Ian Roberts (chair), Haleema Shakur, Tim Coats, Phil Edwards, Beverley Hunt, Maria Ramos.

Data Monitoring and Ethics Committee: Rory Collins (chair), Adrian Grant, John Myburgh, Alex Baxter (independent statistician). For the full list of collaborators please see the online version of this Article and Lancet 2010; 376: 23-32. 


\section{Conflicts of interest}

Members of the Writing Committee declare that they have no conflicts of interest.

\section{Acknowledgments}

The London School of Hygiene and Tropical Medicine supported the core trial coordinating staff during the first year of the trial set-up. Funding for the run-in stage was provided by J P Moulton Charitable Foundation and the BUPA Foundation. A grant-in-aid for purchasing the tranexamic acid and placebo was provided by Pfizer. The main phase of this trial was funded by the UK NIHR Health Technology Assessment programme and will be published in full in the Health Technology Assessment journal series. The views and opinions expressed therein are those of the authors and do not necessarily reflect those of the Department of Health.

\section{References}

1 The CRASH-2 Collaborators. Effects of tranexamic acid on death, vascular occlusive events, and blood transfusion in trauma patients with significant haemorrhage (CRASH-2): a randomised placebo-controlled trial. Lancet 2010; 376: 23-32.

2 Henry DA, Carless PA, Moxey AJ, et al. Anti-fibrinolytic use for minimising perioperative allogeneic blood transfusion. Cochrane Database Syst Rev 2007; 4: CD001886.

3 Levy JH. Antifibrinolytic therapy: new data and new concepts. Lancet 2010; 376: 3-4.

4 Lauer MS, Blackstone EH, Young JB, Topol EJ. Cause of death in clinical research: time for a reassessment? J Am Coll Cardiol 1999; 34: 618-20.
5 Yusuf S, Wittes J, Probstfield J, Tyroler HA. Analysis and interpretation of treatment effects in subgroups of patients in randomized clinical trials. JAMA 1991; 266: 93-98.

6 Sun X, Briel M, Walter SD, et al. Is a subgroup effect believable? Updating criteria to evaluate the credibility of subgroup analyses. BMJ 2010; 340: c117.

7 Assmann SF, Pocock SI, Enos LE, Kasten LE. Subgroup analysis and other (mis)uses of baseline data in clinical trials. Lancet 2000 355: 1064-69.

8 Brohi K, Cohen MJ, Ganter MT, et al. Acute coagulopathy of trauma: hypoperfusion induces systemic anticoagulation and hyperfibrinolysis. J Trauma 2008; 64: 1211-17.

9 Hess JR, Brohi K, Dutton RP, Hauser CJ, Holcomb JB, Kluger Y. The coagulopathy of trauma: a review of mechanisms. J Trauma 2008; 65: 748-54.

10 Sawamura A, Hayakawa M, Gando S, et al. Disseminated intravascular coagulation with a fibrinolytic phenotype at an early phase of trauma predicts mortality. Thromb Res 2009; 1214: 608-13.

11 Prentice CR. Basis of antifibrinolytic therapy. J Clin Pathol 1980; 33: $35-40$

12 Roberts I, Shakur H, Ker K, Coats T, for the CRASH-2 Trial collaborators. Antifibrinolytic drugs for acute traumatic injury. Cochrane Database Syst Rev 2011; 1: CD004896.

13 NIHR Health Technology Assessment Programme. Development and validation of a risk score for trauma patients with haemorrhage. August 2010. http://www.hta.ac.uk/protocols/200900220165.pdf (accessed March 6, 2011) 
CRASH-2 trial collaborators by country

Albania (115)—National Trauma Centre Hospital: Fatos Olldashi, Mihal Kerçi, Tefik Zhurda, Klotilda Ruçi; Spitali Civil Durres: Arben Banushi. Argentina (51)_Hospital Ángel Cruz Padilla: Mario Sardón Traverso, Juan Jiménez; Hospital Regional Rio Grande: Jorge Balbi; Hospital "4 de Junio" Dr Ramon Carrillo: Christian Dellera; Hospital Castro Rendón: Silvana Svampa; Hospital San Martín de La Plata: Gustavo Quintana; Hospital Municipal de Agudos "Dr Leonídas Lucero": Gustavo Piñero; Hospital Interzonal General de Agudos "Dr Oscar Alende": Jorge Teves. Australia (17)-Nepean Hospital: Ian Seppelt; Sir Charles Gairdner Hospital: David Mountain; John Hunter Hospital: Zsolt Balogh. Bangladesh (12)_United Hospital Limited: Maniruz Zaman. Belgium (51)—Sint-Vincentius Hospital: Patrick Druwé, Robert Rutsaert; Centre Hospitalier Regional de Namur: Guy Mazairac. Cameroon (124)-Tombel District Hospital: Fogang Pascal, Zognou Yvette, Djeuchon Chancellin; St Theresa's Catholic Medical Centre: Patrick Okwen; Bamenda Provincial Hospital: Jules Djokam-Liapoe; Bali District Hospital: Ernest Jangwa; Bafut District Hospital: Lawrence Mbuagbaw; Fundong District Hospital: Ninying Fointama; St John of God Medical Centre: Fogang Pascal. Canada (2)-Hamilton General Hospital: Frank Baillie.

China (51)-Renji Hospital: Ji-yao Jiang, Guo-yi Gao, Yin-hui Bao. Colombia (2940)-University of Antioquia, Hospital San Vicente de Paul: Carlos Morales, Juan Sierra, Santiago Naranjo, Camilo Correa, Carolina Gómez; Hospital Universitário San Jose de Popayan: Jorge Herrera, Liliana Caicedo, Alexei Rojas, Henry Pastas, Hugo Miranda; Hospital Pablo Tobon Uribe: Alfredo Constaín, Mayla Perdomo, Diego Muñoz, Álvaro Duarte, Edwin Vásquez; Hospital San Andrés de Tumaco: Camilo Ortiz, Bernardo Ayala, Hernán Delgado, Gloria Benavides, Lorena Rosero; Fundación Clínica Valle del Lili: Jorge Mejía-Mantilla, Ana Varela, Maríaisabel Calle, José Castillo, Alberto García; Clínica las Americas: Juan Ciro, Clara Villa, Roberto Panesso; Hospital General de Medellin: Luz Flórez, Argemiro Gallego; Hospital San Felix ESE: Fabián Puentes-Manosalva, Leonor Medina, Kelly Márquez; Hospital Universitário del Caribe:

Adalgiza Reyes Romero, Ricardo Hernández, Julio Martínez; Hospital Universitário San Jorge: Wilson Gualteros; Hospital San Rafael Tunja: Zulma Urbina, Julio Velandia; Clínica La Estancia SA: Federico Benítez, Adolfo Trochez; Fundación Hospital San José de Buga: Andrés Villarreal, Pamela Pabón; Hospital Civil de Ipiales: Hernán Delgado; Hospital Universitário Departamental Nariño: Héctor López; Hospital Universitário del Valle: Laureano Quintero; Hospital Universitário de Neiva: Andrés Rubiano; Hospital Manuel Uribe Ángel: Juan Tamayo. Cuba (575)—Hospital Clínico-Quirúrgico Docente "Saturnino Lora": Marjoris Piñera; Hospital General Universitário "Carlos Manuel de Céspedes": Daimi Martínez, Heriberto Martínez; Hospital Provincial Docente "Manuel Ascunce Domenech": Eugenio Casola; Hospital Universitário "Arnaldo Milián Castro": Mario Domínguez, Carlos Herrera; Hospital Universitário "Dr Gustavo Aldereguía Lima": Marcos Iraola, Omar Rojas; Hospital Abel Santamaría Cuadrado: Irene Pastrana, Daniel Rodríguez, Sergio Álvarez de la Campa; Hospital Miguel Enríquez: Thorvald Fortún; Hospital General Calixto García: Martha Larrea; Hospital Antonio Luaces Iraola: Lensky Aragón; Hospital Provincial Docente VI Lenin: Aida Madrazo. Czech Republic (17)-Research Institute for Special Surgery and Trauma: Petr Svoboda. Ecuador (1198)—Hospital Luis Vernaza: Mario Izurieta, Alberto Daccach, Mónica Altamirano, Antonio Ortega, Bolívar Cárdenas, Luis González; Hospital José Carrasco Arteaga: Marcelo Ochoa, Fernando Ortega, Fausto Quichimbo, Jenny Guiñanzaca; Hospital de Niños Dr Roberto Gilbert Elizalde: Ines Zavala, Sayra Segura; Hospital Naval Guayaquil: Johnny Jerez; Hospital Alcivar: Daniel Acosta; Hospital

"Dr Rafael Rodríguez Zambrano": Fabián Yánez; Clínica De Especialidades Medicas "San Gregorio": Rubén Camacho. Egypt (2234)-Mataria Teaching Hospital: Hussein Khamis, Hossam Shafei, Ali Kheidr, Hani Nasr, Moetaz Mosaad, Safwat Rizk; Suez Canal University: Hesham El Sayed, Taha Moati, Emad Hokkam; Aswan Teaching Hospital: Mamdouh Amin, Hany Lowis, Medhat Fawzy, Nabil Bedir, Mohamed Aldars. El Salvador (84)—Hospital Nacional Rosales: Virginia Rodríguez, Juan Tobar, Jorge Alvarenga. Georgia (1783)_Tbilisi State University Clinical Hospital
'I Javakhishvili': Budu Shalamberidze, Elza Demuria, Nikoloz Rtveliashvili, Gocha Chutkerashvili, David Dotiashvili; Tbilisi First Hospital, University Clinic, Neurosurgery Center: Tamar Gogichaishvili, George Ingorokva, David Kazaishvili, Besik Melikidze, Natia Iashvili; Tbilisi City Hospital \#1: Gia Tomadze, Manana Chkhikvadze, Leri Khurtsidze, Zviad Lomidze, Diana Dzagania; Tbilisi State Medical University ER Department: Nikoloz Kvachadze, Giorgi Gotsadze, Vakhtang Kaloiani; Institute of Critical Care Medicine: Nino Kajaia. Ghana (136)—Korle Bu Teaching Hospital: Jonathan Dakubo, Simon Naaeder, Priscilla Sowah; Nyinahin Government Hospital: Adamu Yusuf, Alhaji Ishak; Sogakope District Hospital: Paul Selasi-Sefenu; Methodist Hospital Wenchi: Ballu Sibiri; Effia Nkwanta Regional Hospital: Sampson Sarpong-Peprah; Saint Theresa's Hospital: Theodore Boro. India (4768)—Medical Trust Hospital Kochi: Kanjithanda Bopaiah, Kishore Shetty, Raja Subbiah, Lukman Mulla, Anand Doshi; Christian Medical College Ludhiana: Yashbir Dewan, Sarvpreet Grewal, Pradipta Tripathy, Jacob Mathew, Bharat Gupta; Aditya Neuroscience Centre: Anil Lal, Majulie Choudhury; Sri Sai Hospital: Sanjay Gupta, Smita Gupta, Arun Chug; Care Hospital: Venkataramana Pamidimukkala, Palaniappan Jagannath, Mohan Maharaj, Ramaraju Vommi, Naresh Gudipati; North Bengal Neuro Research Centre: W H Chhang; Sheth VS General Hospital and NHL Municipal College: Pankaj Patel, Nilay Suthar, Deepa Banker, Jyotish Patel; LTM Medical College and General Hospital: Satish Dharap, Ranjeet Kamble, Shraddha Patkar, Sushil Lohiya; Government Medical College and Associated Hospitals Jammu: Rakesh Saraf, Dinesh Kumar, Satish Parihar, Rahul Gupta; MKCG Medical College:

Rasananda Mangual, Alagumuthu, Don Kooper, Chinmaya Mohapatra; Christian Medical College Hospital Vellore: Suresh David,

Wesley Rajaleelan, Appas; KLE Hospital and Medical Research Centre: Ashok Pangi, Vivek Saraf, Santhosh Chikareddy; NKP Salve Institute of Medical Sciences and Lata Mangeshkar Hospital: Sushil Mankar, Anil Golhar, Rahul Sakhare, Nilesh Wagh; Sanjivani Diagnostics and Hospital: Anil Lal, Dhiman Hazarika; Parkar Hospital: Pratyush Chaudhuri; Jeevan Jyoti Hospital and Research Centre: Prakash Ketan; Mansarovar Hospital: Govindbhai Purohit, Yogesh Purohit, Mandakini Pandya; Postgraduate Institute of Medical Science Rohtak: Rakesh Gupta, Shashi Kiran, Saurab Walia; Goyal Hospital Jalna: Sonam Goyal, Sidhant Goyal, Satish Goyal; Government Medical College Chandigarh: Sanjay Gupta, Ashok Attri, Rajeev Sharma; Oberai Hospital: Ashok Oberai, Mahesh Oberai, Supriya Oberoi; Rajeev Gandhi Memorial Hospital and Research Centre: Gajendra Kant Tripathi; Calicut Medical College Hospital: Vijayan Peettakkandy,

Premkumar Karuthillath, Pavithran Vadakammuriyil; Krishnamai Medical and Research Foundation's NIKOP Hospital: Jalindar Pol, Sunita Pol, Manisha Saste; St Stephen's Hospital: Subrat Raul, Shashi Tiwari, Neileino Nelly; Government Rajaji Hospital: M Chidambaram; Medical College Trivandrum:

Viswanathan Kollengode, Sam Thampan; Sanjeevani Hospital: Sunder Rajan, Sushrut Rajan; Kamineni Hospital: Subodh Raju, Renuka Sharma; Sri Sakthi Hospital: Subbiah Venkatesh Babu, Chellappa Sumathi; Bhattacharya Orthopaedic and Related Research Centre: Protyush Chatterjee, Alok Agarwal; Sushrut Hospital: Hemant Magar, Meera Magar; All India Institute of Medical Sciences: Manmohan Singh, Deepak Gupta; GM Hospital (P): LTD: Anil Lal, Kamal Haloi; Government Medical College and Superspeciality Hospital Nagpur: Varsha Sagdeo, Pramod Giri; Government Medical College New Civil Hospital: Nimesh Verma, Ravi Jariwala, Ashish Goti; Chikitsa Hospital: Aman Prabhu-Gaonkar, Sagar Utagi; Apollo Health City: Mahesh Joshi, Ruchit Agrawal; Apex Neurotrauma and Superspeciality Hospital: Gopal Sharma, Gurvinder Saini; Neuro Center Gola Ghat: Vinod Tewari; NSCB Medical College: Yad Yadav, Vijay Parihar; BGS Global Hospital: Neelam Venkataramana, Shailesh Rao; Chettinad Hospital and Research Institute: Narayana Reddy; Sir Sayajirao General Hospital and Medical College Baroda: Virsing Hathila; Goyal Hospital and Research Centre Jodhpur: Vithal Das; Krishna Surgical Hospital and Trauma Care Centre: Kantibhai Agaja; Nizam's Institute of Medical Sciences: Aniruddh Purohit; Niramay Hospital: Akilesh Lahari; Apex Hospital Bhopal: Rajesh Bhagchandani; Dr Jeyasekharan Medical Trust: Bala Vidyasagar; Himalayan Institute of Medical Sciences: P K Sachan; Apollo Gleneagles Hospitals: Tanmoy Das; Civil Hospital Gandhinagar: 
Sharad Vyas; Sukhdev Raj Soin Hospital: Sujoy Bhattacharjee; Sancheti Institute for Orthopaedics and Rehabilitation: Parag Sancheti; St James Hospital: T Manoj; Al Shifa Hospital: Mubarak Moideen; Anant Institute of Medical Sciences: Kailash Pansey; Vinayaka Mission Hospital: V P Chandrasekaran; Gauhati Medical College and Hospital: Kabul Saikia; Krishna Hospital and Medical Research Centre: Hoshedar Tata; Ruby Hall Clinic: Sanjay Vhora; Shreejee Hospital: Aniket Shah; Nazareth Hospital: Gordon Rangad; Ganga Hospital: S Rajasekaran; Vadamalayan Hospitals: S T Shankarlal; Devadoss Multispeciality Hospital: Sathish Devadoss; KIOT Hospital: M Saleem; Baby Memorial Hospital: Haroon Pillay; Bethany Hospital: Zulfiquer Hazarika; Suretech Hospital and Research Centre: Parikshit Deshmukh; Surya Hospital: S P Murugappan; Apollo Clinic Varanasi: Amit Jaiswal; Fortis Escorts Hospital: Deepak Vangani; Gokul Hospital and Trauma Centre: Prakash Modha; International Hospital Assam: Chawngrolien Chonzik; Lifeline Multispeciality Hospital: Megha Praveen; Meenakshi Mission Hospital and Research Centre: Vijaya Sethurayar; MOSC Medical College Hospital: Sojan Ipe; MS Ramaiah Memorial Hospital: Naresh Shetty; Saykhedkar Hospital and Research Centre: Aniket Shah; Shanti Mukand Hospital: R P Gupta; Shri KM Memorial Jain Heart and General Hospital: Vinod Jain; Usha Hospital: Ketan Shah. Indonesia (706)—Soebandi Hospital Jember: Moch Dwikoryanto; Sanglah General Hospital: Nyoman Golden, Kuning Atmadjaya, Ketut Wiargitha, Ketut Sudiasa, Gede Suwedagatha; Saiful Anwar General Hospital: Farhad Bal'afif; Dr Soetomo General Hospital: Vicky Budipramana, Tabrani, Agung Lemuel; Cipto Mangunkusumo Hospital: Susilo Chandra; Muhammadiyah Lamongan Hospital: Faisol Ama. Iran (134)—Nemazi Hospital: Ehsan Sherafatkazemzadeh, Ehsan Moradi, Alireza Sheikhi; Erfan Hospital: Ali Ziaee, Ahmad Fanaei; Loqman Medical Center: Esmaeil Hajinasrollah; Imam Hosain Hospital: Kamran Heidari. Iraq (392) - Diwaniyah College of Medicine: Bassim Mohammad, Najah Hadi. Italy (57)-Spedali Civili di Brescia: Giovanna Perone, Elena de Peri; Azienda Ospedaliera Di Parma: Annalisa Volpi. Jamaica (11)_University Hospital of the West Indies: Jean Johnson. Japan (9)-Fukuoka University Hospital: Masayoshi Abe. Kenya (31)—Kenyatta National Hospital: Vincent Mutiso, Benjamin Okanga; Kapenguria District Hospital: Daniel Ojuka. Malaysia (216) - Hospital University Science Malaysia: Baharudin Abdullah, Hishamuddin Rahman, Yazid Noh; Sungai Buloh Hospital: Sabariah Jamaluddin, Hasnah Dawal; University of Malaya Medical Centre: April Roslani, Chee-Wei Law, P Devashanti; Hospital Tengku Ampuan Rahimah: Yusof Wahab, Shanta Velaiutham; Ampang Hospital: Ridzuan Dato. Mexico (479)—Hospital General Regional 25: Jorge Loría, Erandy Montes, Eduardo Gómez, Víctor Cazales, Paúl Bautista; Hospital Gustavo Rovirosa: Raúl Bautista,

David Ahumada, Edwin Hernández, Germahin Velásquez; Hospital General de Uruapan "Dr Pedro Daniel Martínez": Patricia Ortega, Gabriel Lira, Francisco Estrada; Hospital General Ecatepec Las Américas: Jorge Martínez; Hospital General La Perla: Juan Martínez; Hospital General de Ecatepec "Dr José María Rodríguez": Julio Casasola. Nigeria (2053)—National Hospital Abuja: Oluwole Olaomi, Yari Abubakar, Kanati Apollo, Olawale Badejo, Ogemdi Ihekire; University of Benin Teaching Hospital: Pius Iribhogbe, Olugbenga Oludiran, Emmanuel Obeta, Collins Okojie Ernest Udefiagbon; Obafemi Awolowo University Teaching Hospitals: Edward Komolafe, Patrick Olaleye, Tochukwu Uzochukwu, Uvie Onakpoya; Irrua Specialist Teaching Hospital: Andrew Dongo; Olabisi Onabanjo University Teaching Hospital: Lateef Thanni; University College Hospital Ibadan: Adefemi Afolabi, Titilade Akinola, Adeyinka Ademola, Olusola Akute; Ahmadu Bello University Teaching Hospital: Lawal Khalid, Lawal Abubakar, Muhammad Aminu, Mike Ogirima; Baptist Medical Centre: Ambrose Attansey, Durodola Michael, Olaniran Aremu; University of Ilorin Teaching Hospital: Odebode Olugbenga, Udoffa Ukpong, Yusuf Salman; Enugu State University Teaching Hospital: Nene Obianyo, Chinenye Ani, Roderick Ezeadawi; LAUTECH Teaching Hospital: Oluwadiya Kehinde,
Agodirin Olaide; Federal Medical Centre Makurdi: Andrea Jogo, Terna Bitto; Nnamdi Azikiwe University Teaching Hospital: Stanley Anyanwu, Okechukwu Mbonu; Lagos State University Teaching Hospital: Mobolaji Oludara, Michael Somoye; Usmanu Danfodiyo University Teaching Hospital: Bello Shehu, Nasir Ismail; National Orthopaedic Hospital Enugu: Amechi Katchy; University of Calabar Teaching Hospital: Rowland Ndoma-Egba, Ngim Grace-Inah; University of Abuja Teaching Hospital: Zumnan Songden, Abdulrahman Abdulraheem; University of Uyo Teaching Hospital: Akpan Otu, Timothy Nottidge; Federal Medical Centre, Yenagoa: Domingo Inyang, David Idiapho; Seventh Day Adventist Hospital: Herb Giebel; Federal Medical Centre Birnin-Kebbi: Ramatu Hassan; Abia State University Teaching Hospital: Adeyinka Adisa; Wesley Guild Hospital: Akinbolaji Akinkuolie; Federal Medical Centre, Umuahia: Kalu Okam; University of Maiduguri Teaching Hospital: Abubakar Musa; National Orthopaedic Hospital, Igbobi: Ignatius Falope; University of Nigeria Teaching Hospital Enugu: John Eze. Peru (452)—Hospital Regional Docente de Trujillo: José Caballero, Wenceslao Azabache, Oscar Salirrosas; Hospital Nacional Hipolito Unanue: Alonso Soto, Elfi Torres, Gloria Ramírez, Mónica Pérez; Clinica Santa Ana: Cesar Malca; Hospital La Caleta: Juan Velez; Hospital Nacional Sergio E Bernales: Raul Yepez; Hospital de Apoyo de Sullana: Hernan Yupanqui; Hospital IV Essalud Huancayo: Pedro Lagos; Hospital Nacional Arzobispo Loayza: Diana Rodriguez; Hospital Municipal Los Olivos: Jorge Flores; Hospital Jose Cayetano Heredia: Anselmo Moya; Hospital Nacional Carlos Alberto Seguin Escobedo: Alejandro Barrionuevo; Hospital Nacional Dos De Mayo: Marco Gonzales-Portillo; Hospital Nacional Cayetano Heredia: Edgar Núñez. Saudi Arabia (70)—King Khalid University Hospital: Abdelazeem Eldawlatly, Mohammed Al Naami, Bilal Delvi; King Khalid National Guard Hospital: Walid Alyafi. Serbia (1)—Klinicki Centar Srbije: Branko Djurovic. Singapore (2)—National Neuroscience Institute: Ivan Ng. Slovakia (38)_FNsP Ružinov: Aktham Yaghi; NsP Poprad: Anton Laincz; NsP JA Reiman Hospital: Stefan Trenkler; Faculty Hospital F D Roosevelta: Jozef Valky. South Africa (76)-Dr George Mukhari Hospital: Mphako Modiba, Peter Legodi, Thomas Rangaka; George Provincial Hospital: Lee Wallis. Spain (23)—Hospital Universitário Virgen del Roció: Ángeles Muñoz; Hospital Ramón y Cajal de Madrid: Ana Serrano; Hospital Universitário Germans Trias i Pujol: Pilar Marcos; Hospital Torrecardenas: Martin Rubi; Hospital Universitário Virgen de la Victoria: Victoria de la Torre. Sri Lanka (103)—National Hospital of Sri Lanka: Ranjith Ellawala, Samitha Wijeratna, Lukshrini Gunaratna, Crishantha Wijayanayaka. Tanzania (64)—Muhimbili Orthopaedic Institute: Kitugi Nungu, Billy Haonga, Grenda Mtapa. Thailand (903)—Khon Kaen Regional Hospital: Surakrant Yutthakasemsunt, Warawut Kittiwattanagul, Parnumas Piyavechvirat, Tawatcahi Impool, Santipong Thummaraj; Pattani Hospital: Rusta Salaeh; Suratthani Hospital: Sakchai Tangchitvittaya; Bhumibol Adulyadej Hospital:

Kamol Wattanakrai, Teerasak Jiravongbunrod, Chatchai Soonthornthum; Lampang Hospital: Surasak Meephant; Rayong Hospital:

Pusit Subsompon; Roi-Et Hospital: Phaiboon Pensuwan; Phrae Hospital: Wicheanrat Chamnongwit. Tunisia (36)-Hospital Habib Thameur: Zouheir Jerbi, Abderraouef Cherif. UK (135)-University Hospital of North Staffordshire: Mark Nash; Royal London Hospital: Tim Harris; Leicester Royal Infirmary: Jay Banerjee; Nottingham University Hospitals NHS Trust: Ramzi Freij; Frenchay Hospital: Jason Kendall; Countess of Chester Hospital: Stephen Moore; Hull Royal Infirmary: William Townend; Royal Sussex County Hospital: Rowland Cottingham; Derby Hospitals NHS Trust: Dan Becker; Bedford Hospital NHS Trust: Stuart Lloyd; Royal Liverpool University Hospital: Peter Burdett-Smith; Colchester General Hospital: Kazim Mirza; Royal Lancaster Infirmary: Andrew Webster; Worthing Hospital: Suzanne Brady, Amanda Grocutt; Darent Valley Hospital: John Thurston; Hope Hospital: Fiona Lecky; Northern General Hospital: Steve Goodacre. Zambia (62)-University Teaching Hospital, Lusaka: Yakub Mulla, Dennis Sakala; Nchanga North General Hospital: Charles Chengo. 